Lequoy P, Savoji H, Saoudi B, Bertrand-Grenier A, Wertheimer MR, De Crescenzo G, Soulez G, Lerouge $\mathrm{S}^{*}$. In vitro and pilot in vivo evaluation of a bioactive coating for stent-grafts based on chondroitin sulfate and epidermal growth factor. Journal of Vascular and Interventional Radiology 27 (5): 753-760, 2016.

\title{
In vitro and pilot in vivo evaluation of a bioactive coating for stent-grafts based on chondroitin sulfate and epidermal growth factor
}

Pauline Lequoy MSc, ${ }^{\text {a,b }}$ Houman Savoji MSc, ${ }^{\text {a,c }}$ Bachir Saoudi PhD, ${ }^{\text {a,b }}$ Antony BertrandGrenier MSc, ${ }^{\text {a }}$ Michael R. Wertheimer PhD, ${ }^{\mathrm{c}}$ Gregory De Crescenzo PhD, ${ }^{\mathrm{d}}$ Gilles Soulez MD MSc ${ }^{\mathrm{a}, \mathrm{e}, \mathrm{f}}$ and Sophie Lerouge $\mathrm{PhD}^{\mathrm{a}, \mathrm{b}, \mathrm{e}} *$

\section{Affiliations}

a Research Centre, Centre Hospitalier de l’Université de Montréal (CRCHUM), 900 rue Saint Denis, Montréal (Qc), Canada H2X 0A9

b Department of Mechanical Engineering, École de technologie supérieure (ÉTS), 1100 boul. Notre-Dame Ouest, Montréal (Qc), Canada H3C 1K3

C Department of Engineering Physics, École Polytechnique de Montréal, P.O. Box 6079, succ. Centre-Ville, Montréal (Qc), Canada H3C 3A7

d Department of Chemical Engineering, École Polytechnique de Montréal, P.O. Box 6079, succ. Centre-Ville, Montréal (Qc), Canada H3C 3A7

e Department of Radiology, Radio-oncology and Nuclear Medicine, University of Montreal, Pavillon Roger-Gaudry 2900, boul. Édouard-Montpetit, Montréal (Québec) H3T 1J4QC

f Department of Radiology, CHUM, Montreal, Canada 


\begin{abstract}
Purpose: To evaluate the potential of a bioactive coating based on chondroitin sulfate (CS) and tethered epidermal growth factor (EGF) for the improvement of healing around stent-grafts (SG).
\end{abstract}

Methods: The impact of the bioactive coating on cell survival was tested in vitro on human vascular cells using polyethylene terephtalate films (PET) as a substrate. After being transferred onto a more "realistic" material (expanded poly(tetrafluoroethylene), ePTFE), the durability and mechanical behavior of the coating in addition to cell survival were studied. Preliminary in vivo testing was performed in a canine iliac aneurysm model reproducing type I endoleaks (3 animals with 1 control and 1 bioactive SG for each).

Results: The CS and EGF coatings significantly increased survival of human smooth muscle cells and fibroblasts, compared with bare PET or ePTFE $(P<.05)$. The coating also displayed good durability over 30 days according to ELISA and cell-survival tests. The coating did not affect the mechanical properties of ePTFE and was successfully transferred onto commercial SG for in vivo testing. According to CT-scan and macroscopic examinations, no difference was observed in endoleak persistence at three months, but the bioactive coating deposited on the abluminal surface of the SG (exposed to the vessel wall) increased the percentage of healed tissue in the aneurysm. Moreover, no adverse effect such as neointima formation or thrombosis was observed.

Conclusion: The bioactive coating promoted in vitro cell survival, displayed good durability and was successfully transferred onto a commercial SG. Preliminary in vivo results suggest improved healing around bioactive SG. 


\section{Introduction}

Poor healing is observed around stent grafts (SG) used for the endovascular treatment of abdominal aortic aneurysms (AAA)[1], with a lack of organized tissue formation in the cavity and absence of incorporation of the polymer graft into the surrounding wall of the blood vessel [1, 2]. This can be related to three main factors: (i) the pro-apoptotic environment of a diseased aneurysmal vessel, characterized by changes in extracellular matrix composition, presence of inflammatory cells, vascular cell depletion [3, 4]; (ii) the lack of oxygen and nutrients after SG insertion; and (iii) the nature of the materials used for SG cover, namely polyethylene terephtalate (PET) or poly(tetrafluoroethylene) (PTFE). The deficit of tissue formation on the SG abluminal side could play a role in the occurrence of type I endoleaks and endotension (defined by a sac enlargement of more than $5 \mathrm{~mm}$ without visible leak) that affect respectively 13 and $18 \%$ of patients treated by SG. [5, 6] Presence of bare stent, hooks or barbs at proximal neck helps preventing SG migration, which is observed in 1 to $3 \%$ of cases, by providing strong mechanical attachment to the vessel $[2,5,6]$. However, they do not promote complete biological seal at proximal and distal necks and thus cannot prevent neck dilatation associated with delayed endoleak $[7,8]$.

We hypothesize that modification of SG surface properties via the immobilization of biomolecules could counter aneurysmal degeneration and promote healing around the abluminal side of the SG, thereby preventing late occurence of type I endoleaks and migration of SG through biological seal. The general aims of our work were to develop a bioactive coating that can counter the pro-apoptotic environment observed in the aneurysm sac while promoting colonization of the implant by SMC and fibroblasts, and 
to demonstrate the potential of such a coating to improve tissue growth at the SG external surface.

\section{Materials and Methods}

Two different bioactive molecules were selected to create a bioactive coating: (i) chondroitin sulfate (CS), a glycosaminoglycan of the extracellular matrix that participates in the integrity of the arterial wall in normal aortas [9] and exhibits anti-apoptotic properties [10]; and (ii) epidermal growth factor (EGF), a major actor of wound-healing [11] presenting both anti-apoptotic and pro-proliferative properties [12, 13]. This choice is based on previous studies that have shown the superiority of the combination of CS and EGF [14]. The coating was created on polyethylene terephtalate (PET) and expanded polytetrafluoroethylene (ePTFE) substrates (since these are the two plastic materials used in SG graft fabric) and tested in vitro for its bioactivity on vascular cells and its stability. Then, in vivo testing on a canine iliac aneurysm model was performed for its ability to induce sustained healing in the aneurysmal cavity [15] when coating the external surface of an expanded PTFE-based commercial SG.

\section{Bioactive coating preparation}

Before grafting CS and EGF covalently, primary amine $\left(\mathrm{NH}_{2}\right)$ groups were created on the polymer substrates by means of a 100 nm-thick coating of $\left(\mathrm{NH}_{2}\right)$-rich plasma polymer, here designated as LP, which was deposited using a low-pressure radiofrequency glow discharge reactor [16]. Then, the immobilization of CS and EGF on LP surfaces was 
achieved through carbodiimide chemistry using 1-Ethyl-3-(3dimethylaminopropyl)carbodiimide (EDC) and N-Hydroxysuccinimide (NHS) (SigmaAldrich, Oakville, ON).[17] Surfaces were exposed to a $1 \%$ w/v chondroitin-4-sulfate (CS from bovine trachea, Sigma-Aldrich, Oakville, ON) solution containing $40 \% \mathrm{v} / \mathrm{v}$ ethanol, $50 \mathrm{mM}$ MES, $22.8 \mathrm{mM}$ EDC, and $4.6 \mathrm{mM}$ NHS for 1h. After PBS and water rinsing, CS-modified surfaces were disinfected by immersion in $70 \% \mathrm{v} / \mathrm{v}$ ethanol for 5 min. Then, CS carboxyl groups were activated by a solution containing the same concentration of ethanol, EDC and NHS as before. After rinsing, a $100 \mathrm{nM}$ aqueous solution of recombinant human EGF (PeproTech, Rocky Hill, NJ) was incubated on CSmodified surfaces for $1 \mathrm{~h}$.

Chemical and Mechanical Characterization

Surface composition of the coating on ePTFE was characterized using X-ray photoelectron spectroscopy (XPS, VG ESCALAB 3MkII instrument). The wettability of the various coatings was assessed by static water contact angle (WCA) measurements, using Milli-Q water (VCA OptimaXE, AST products). Scanning electron microscopy (SEM, Hitachi S-3600N) was used to evaluate whether coating deposition altered the surface morphology and porosity of the ePTFE substrate. Finally, traction tests were carried out using a Bose Electroforce 3200 system (Eden Prairie, MN) with a deformation rate of $5 \% / \mathrm{s}$, in order to confirm that coating deposition did not affect the mechanical properties (Young's modulus, E, and tensile strength) of ePTFE. 


\section{Biological characterization}

\section{Cell culture materials.}

Human aortic smooth muscle cells (SMC), human aortic adventitial fibroblasts and their respective complete growth media were purchased from Lonza (Walkersville, MD). For conditions designated as 'EGF solution', samples with LP/CS coatings were placed in contact with serum-free culture medium containing $10 \mathrm{ng} / \mathrm{mL}$ of EGF (renewed every 2 days). For all cell culture experiments, tissue culture polystyrene (TCPS) plates were used as controls (Corning Inc., Corning, NY).

\section{Cell survival on PET films.}

Cells were seeded on bare and coated PET films (50 $\mu \mathrm{m}$ thick, Goodfellow, Huntingdon, England), at 40000 cells/ $\mathrm{cm}^{2}$ in complete growth medium. 24h after initial cell seeding, the surfaces were washed with PBS and serum-free medium was added. At each timepoint, cell metabolic activity was probed using the alamarBlue assay (Invitrogen, Burlington, ON). Cell density and homogeneity were also evaluated via crystal violet staining, followed by image capture.

\section{SMC survival on ePTFE membranes.}

To assess cell survival on the more realistic ePTFE membranes (100 $\mu$ m thick, Zeus, Orangeburg, SC), direct counting of nuclei was performed. SMC were left to adhere on ePTFE discs for 24h. The surfaces were rinsed with PBS and serum-free medium was added. After 4 and 7 days, nuclei were stained.[17] Three images were captured for each 
sample using fluorescence microscopy, and cells were counted on each image. After 7 days, cells were fixed in paraformaldehyde and stained,[18] before observation via confocal microscopy (Olympus FV1000MPE).

\section{Durability of the coatings}

The EGF surface density of LP/CS/EGF coatings on ePTFE was evaluated by direct ELISA immediately after preparation, or after 1 day, 1 week and 1 month under three different storage conditions (dry, at room temperature, $\mathrm{RT}$, or $4^{\circ} \mathrm{C}$; and in $\mathrm{PBS}$ at $37^{\circ} \mathrm{C}$ ) using an ELISA kit (R\&D Systems, Minneapolis, MN). In addition, the bioactivity of the coating after 1 month was directly tested by cell survival experiments.

\section{Coating transfer onto stent-grafts}

The bioactive LP/CS/EGF coating was created on commercial SG from Atrium Medical Corp., which consists of a tubular structure formed by two ePTFE layers encapsulating a stainless steel stent (Advanta V12, 6 mm diameter, 59 mm length, Atrium Medical Corp., Hudson, NH). Atrium SG were deployed using a balloon catheter, then inserted on top of a rotatable ceramic cylinder in the plasma deposition chamber. The coating was created as described above, so as to obtain a uniform $150 \mathrm{~nm}$ coating thickness on the outer surface. CS and EGF were successively grafted by immersion in the solutions just prior SG implantation under a sterile hood. The SGs were disinfected by immersion in EtOH $70 \% \mathrm{v} / \mathrm{v}$ for 5 minutes between CS and EGF grafting. Coated and control SG were left to dry under a sterile hood and placed in sterile tubes for transportation to the operation room. 


\section{In vivo implantation procedure}

Preliminary in vivo testing of LP/CS/EGF coatings was performed in a canine bilateral iliac aneurysm model that reproduces persistent type I endoleaks after EVAR.[15] All procedures were approved by the institutional animal committee, in accordance with the guidelines of the Canadian Council on Animal Care, and performed under general anesthesia. Three mongrel dogs weighing 25-30 kg were subjected to surgical construction of bilateral iliac aneurysms without collateral, using an autologous jugular vein patch as already described in details.[16]

After a recovery period of 8 weeks, the SG was inserted by a carotid approach under fluoroscopy, and expanded to 110\% of the nominal arterial diameter (median $6 \mathrm{~mm}$ ). In each dog, a LP/CS/EGF-modified SG was randomly implanted in one aneurysm, a control (unmodified) SG being implanted in the contralateral aneurysm. On both sides, plastic deformation of the SG was created at its proximal end by inflating a $3.5 \mathrm{~mm}$ diameter Savvy balloon catheter (Cordis Corp.) alongside the SG.[15] This formed a type I endoleak in all aneurysms, with inflow occurring through the misfit ( 1-2 mm gap) at the proximal neck, as confirmed by angiography.

After 3 months, percutaneous transfemoral angiography (Koordinat 3D II, Siemens) and CT-scan (Sensation 64, Siemens) were performed to evaluate the presence and types of endoleaks. The dogs were then sacrificed by barbiturate overdose; the aneurysms were 
fixed in buffered formalin, frozen in liquid nitrogen, and cut into $5 \mathrm{~mm}$-thick transverse sections (Exakt cutting system, Exakt Gmbh, Norderstedt, Germany) taking care to preserve the interface between the tissue and SG. On macroscopic sections, the sizes of areas corresponding to a leak, to healed tissue (organized tissue rich in cells and collagen), and to fresh thrombus (coagulated blood showing absence or poor cell organization) were calculated after segmentation and expressed as a percentage of the total area. Some sections were sent for conventional histology after careful removal of metallic SG parts and stained with hematoxylin phloxine saffron (HPS) and Movat. Other sections containing the SG were dehydrated, embedded in acrylic resin (Technovit 7200; Heraeus-Kulzer, Armonk, NY, USA), cut, and polished into $30 \mu \mathrm{m}$ sections that were then stained with hematoxylin and eosin.

\section{Statistical analysis}

Results are expressed as mean \pm standard deviation. Statistics were assessed by one-way ANOVA with Tukey's HSD post-hoc testing. For continuous variables measured in aneurysms implanted with active and control SGs, a paired student t-test with equal variances was used, $\mathrm{p}$-values less than 0.05 being considered significant.

\section{Results}

\section{Coating characterization and stability}

As expected, LP deposition on PTFE led to an increase in nitrogen content on the surface (Table 1$)$ and strongly increased hydrophicility $\left(84.6 \pm 4.4^{\circ}\right.$ for the water contact angle, 
instead of $115.8 \pm 2.4^{\circ}$ ). The absence of fluorine in the XPS spectra after LP deposition confirmed that the LP layer was homogenous and thick enough to completely mask the underlying ePTFE substrate. CS immobilization on LP was confirmed by XPS via increase in surface content of sulfur, and by a further increase in hydrophicility $\left(62.9 \pm 5.0^{\circ}\right)$. EGF grafting on the surface was confirmed by ELISA and was found to remain stable over 1 month under all storage conditions, with $102 \pm 18 \%, 119 \pm 42 \%$ and $89 \pm 20 \%$ of initial signal remaining after aging for 30 days under dry conditions at room temperature, dry conditions at $4^{\circ} \mathrm{C}$ or in $\mathrm{PBS}$ at $37^{\circ} \mathrm{C}$, respectively (Fig. 1). This indicated that the EGF was still attached to the surface and that the epitope of the EGF molecule that reacted with the EGF antibody was preserved. Bioactive coating deposition did not affect mechanical properties and morphology of the underlaying ePTFE, according to SEM observations and tensile tests (Fig. E1 and E2, available online at www.jvir.org)

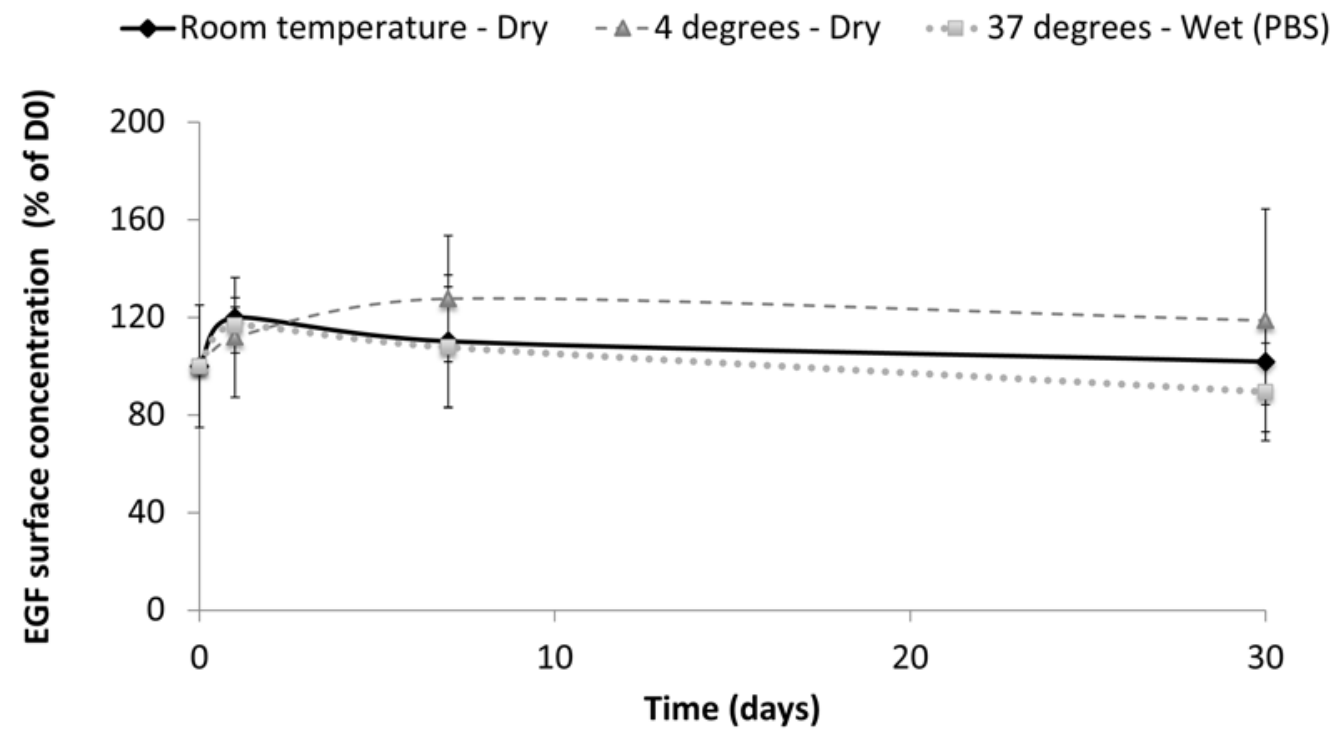

Fig. 1. ELISA tests confirmed the presence of EGF in the coating and its stability over a one-month storage period. EGF concentration was measured immediately after 
coating and after 1 day, 1 week and 1 month storage, in dry conditions at room temperature (black diamonds, solid line) or at $4^{\circ} \mathrm{C}$ (dark grey triangles, dotted line), or in wet conditions (PBS, phosphate buffer solution) at $37^{\circ} \mathrm{C}$ (light grey squares, dots). Data are normalized to the value obtained immediately after grafting.

Cell adhesion and survival on coated ePTFE

SMC initially adhered equally well on all surfaces except on bare ePTFE, where the adhesion was significantly lower $(P<.05$, Fig. 2$)$. The bioactive LP/CS/EGF coating significantly increased cell survival compared with bare ePTFE $(P<.05)$, as shown by the number of cells after 4 and 7 days in serum-free medium. Interestingly, EGF covalently grafted on CS led to similar cell survival as EGF added in solution on LP/CS surfaces, with a constant cell number over 7 days. Simple coating by LP and LP/CS also improved cell survival compared with ePTFE, but this positive effect did not last over 7 days.

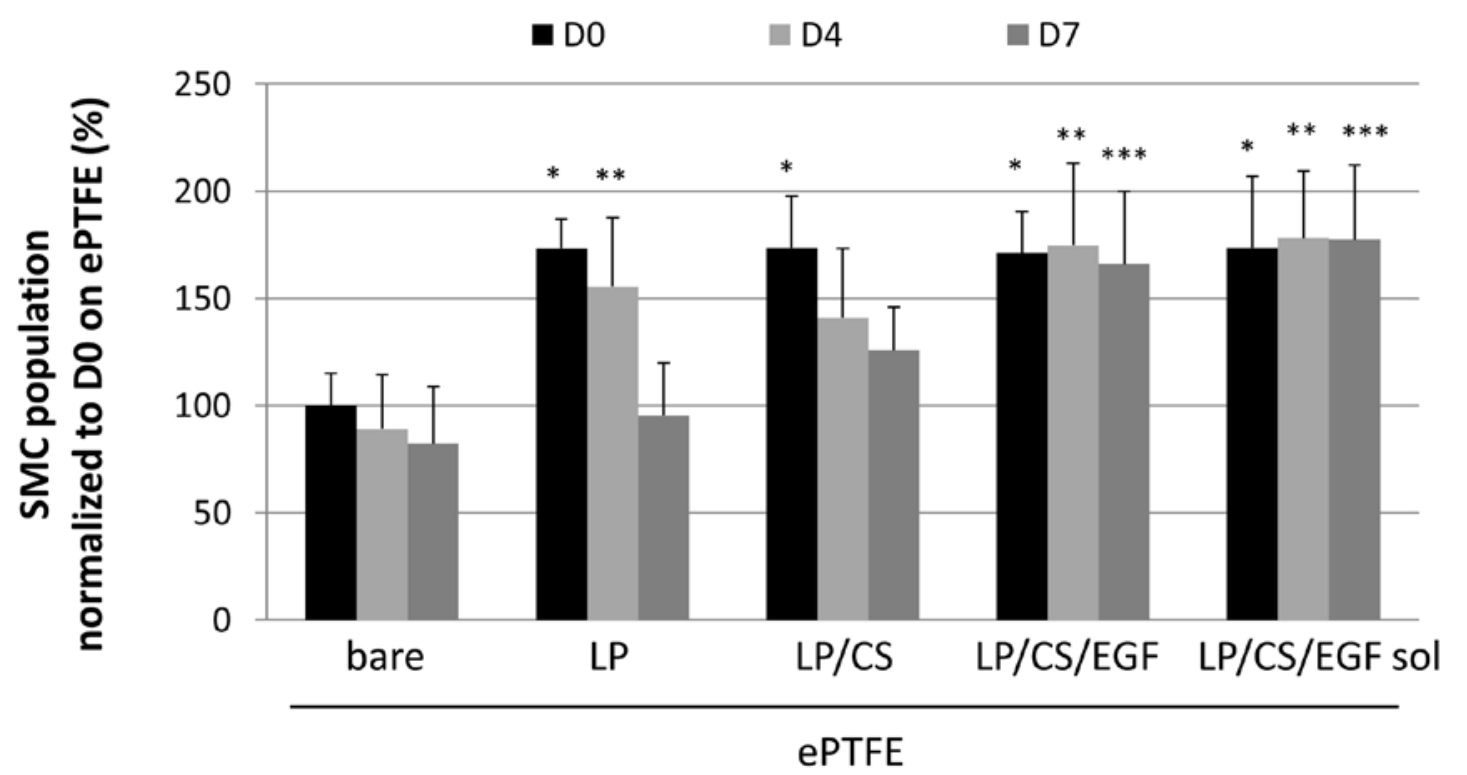

Fig. 2. The bioactive coating significantly increased cell survival compared to bare ePTFE. SMC adhesion (D0) and survival in serum free medium for 4 and 7 days (D4, D7) was estimated by cell count after Hoescht 33342 staining and normalized to the cell count on ePTFE at D0 for each experiment. *, **,***: $P<.05$ compared to bare ePTFE at D0, D4 and D7 respectively $(n=12)$. ePTFE: expanded polytetrafluoroethylene, LP/CS/EGF: complete bioactive coating, LP: primary amine-rich plasma polymer 
coating, LP/CS: chondroitin sulfate grafted on LP, LP/CS/EGFsol: surface modified by $L P+C S$ and EGF added in solution at $10 \mathrm{ng} / \mathrm{ml}$.

Cell morphology was drastically different on bare ePTFE compared with all coated surfaces. The cells formed large clusters that favored cell-cell over cell-substrate interactions (Fig. 3). This explains the stable number of cells on ePTFE, despite their evident lack of interaction with the hydrophobic surface. In contrast, cells were well spread and homogeneously distributed on coated surfaces.
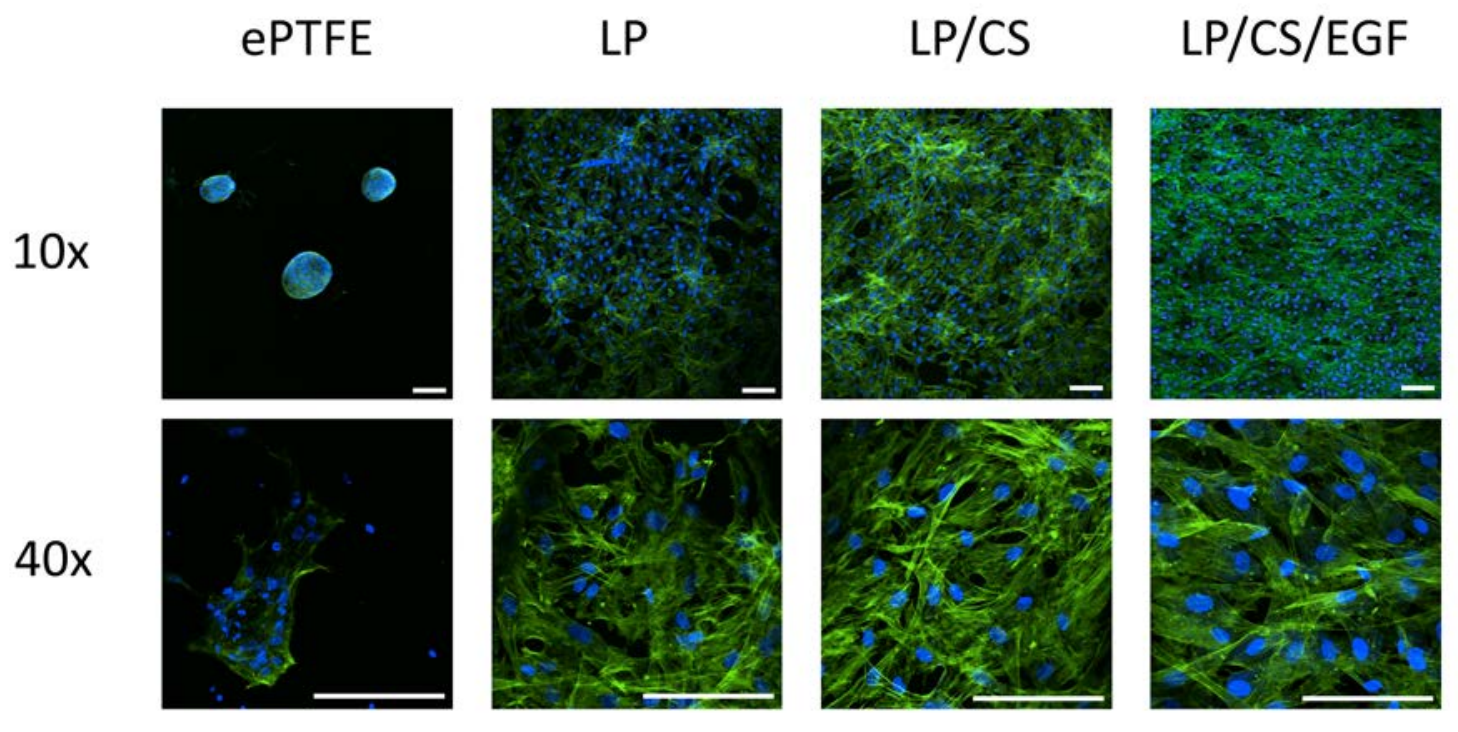

Fig. 3. Confocal microscope images of SMC after 7 days. Nuclei was stained with Hoescht 33342 (blue) and actin cytoskeletons with phalloidin-Alexa 488 (green). Cell morphology was drastically different on bare vs. modified material: cells formed large clusters on ePTFE, while they were well spread and homogeneously distributed on coated surfaces. Scale bars: $150 \mu \mathrm{m} .(n=4)$.

Cell survival tests performed on coatings stored for 1 month showed similar results as fresh coatings. Cell survival after 7 days in serum-free medium was $146 \pm 55 \%$ after storage in air at RT, $155 \pm 39 \%$ after storage at $4^{\circ} \mathrm{C}$, and $165 \pm 49 \%$ after storage in PBS 
at $37^{\circ} \mathrm{C}$, compared with $166 \pm 62 \%$ on fresh surfaces $(\mathrm{p}>0.5)$. This confirms the stability of the coating.

Significantly higher cell survival was also demonstrated, with both SMC and fibroblasts, on coated PET substrates compared with unmodified controls (Fig. E3 and E4; available online at www.jvir.org)

\section{In vivo study}

A homogeneous coating was then successfully created on the commercial SG (Figure E5) before in vivo implantation in iliac aneurysms. Three months after implantation, no SG migration was observed, and all SG remained patent, as confirmed by CT-scan. Endoleaks, characterized by well-defined empty areas on histology slides and bright areas on contrast-enhancement CT-scan (Fig. 4a and Fig.E6), persisted in all aneurysms at three months. However, the portion of aneurysm corresponding to healed tissue was significantly greater in the presence of LP/CS/EGF coating (Fig. 5, $P<.02$ ), and portions consisting of leak or fresh thrombus were lower for those aneurysms that were treated with the bioactive SG (Fig. 4b). However, no definite conclusion should be drawn, since the number of samples was small $(n=3)$ and results varied significantly from one animal to another. 
a.
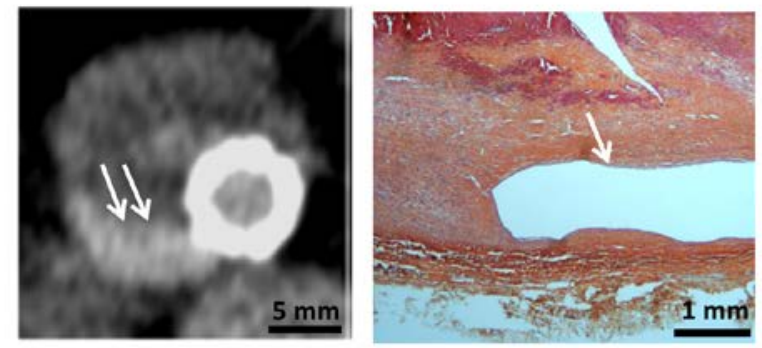

b.
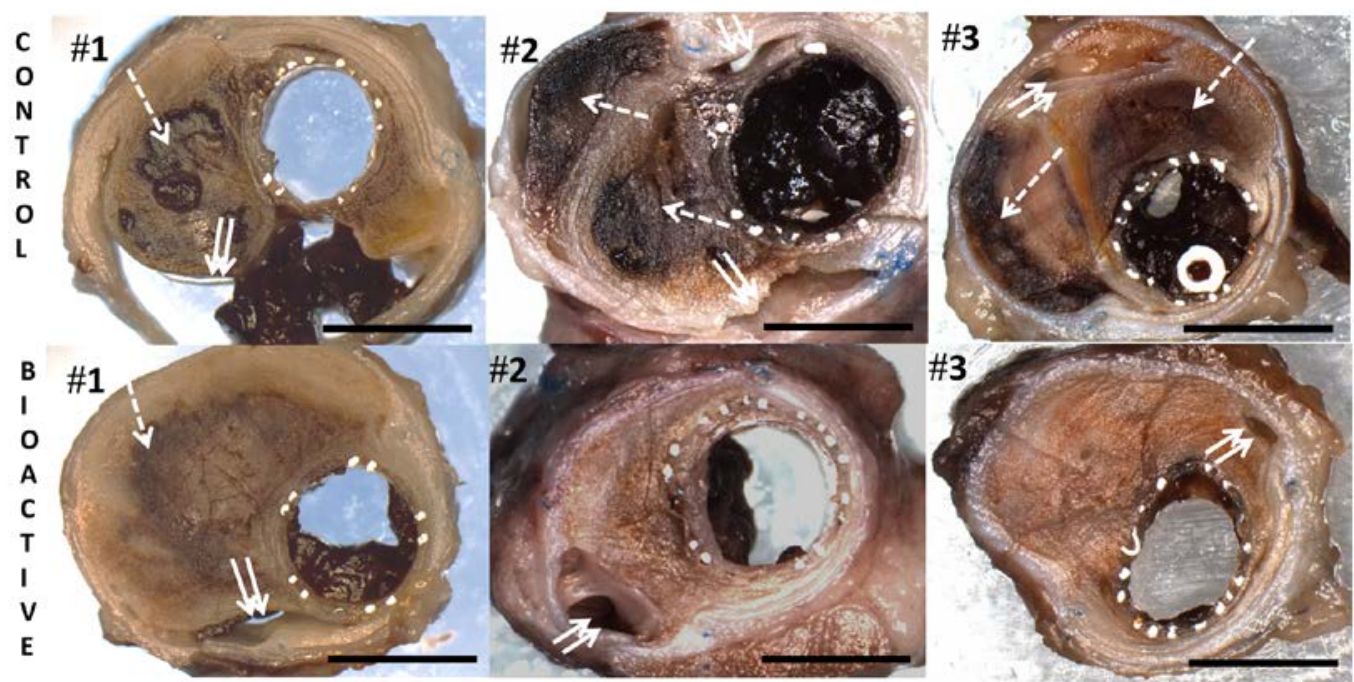

Fig. 4 (a) Example of endoleaks from dog \#1, bioactive SG, detected on CT-Scan (left) and histological section stained by HPS (right). (b) Transverse tissue sections taken at the middle of aneurysms for each dog, with control (first row) or bioactive SG (second row). Leak are indicated by double arrows and fresh thrombus by single dotted arrows. Scale bars: $5 \mathrm{~mm}$. n.b. some post-mortem intraluminal thrombus is present in the lumen. Bioactive SG displayed visibly smaller leaks and less fresh thrombus compared to control SG. 


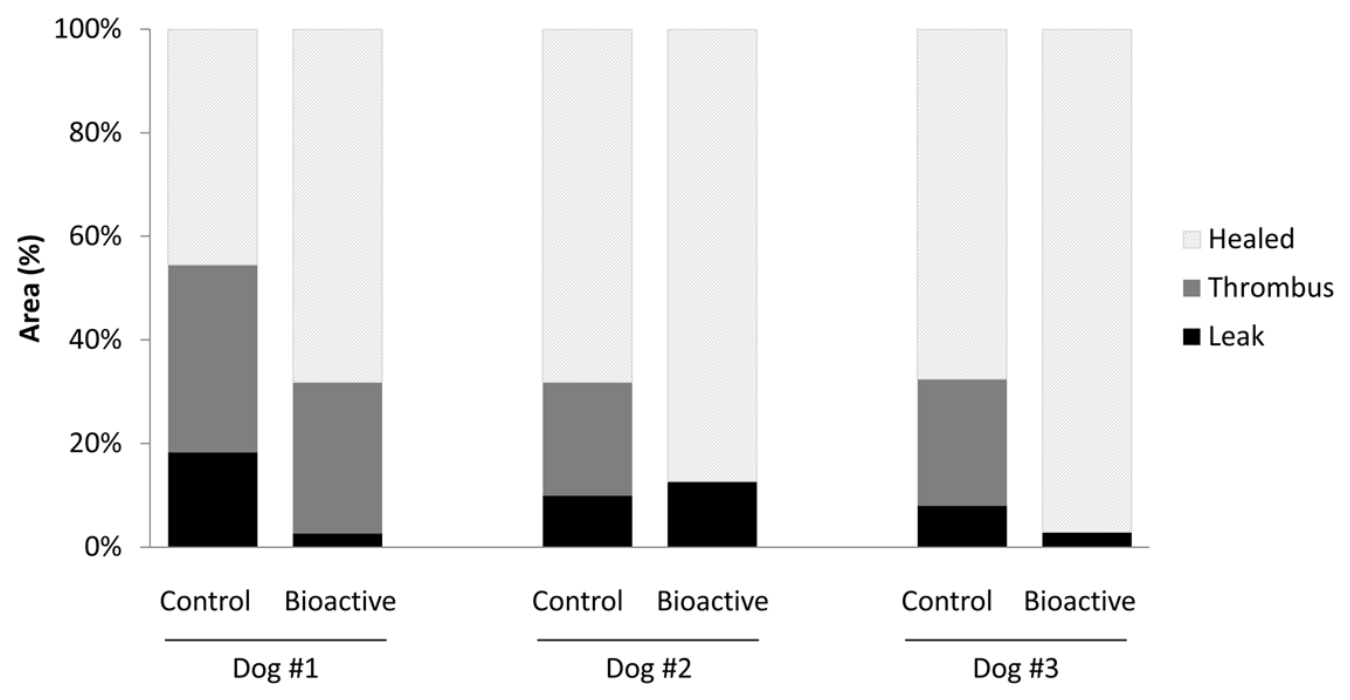

Fig. 5 Maximal area (in percentage of the total aneurysm section) corresponding to leak and fresh thrombus for the 3 bioactive and 3 control SG. The proportion of aneurysm corresponding to healed tissue was significantly higher in the presence of the bioactive coating.

Of interest, observations of explanted SG did not show increased neointimal hyperplasia or intraluminal thrombus on the lumen side of the bioactive SG compared with bare ePTFE SG (intraluminal neointima thickness at the proximal neck was $<5 \%$ compared with the luminal diameter for all SG).

\section{Discussion}

Recently, several non-bioactive devices have been developed to prevent endoleaks (including type I), using polymer-injectable structures that either provide on-site fitting at the proximal neck,[19] or that fill the whole cavity [20]. While these structures may allow better initial sealing and adaptation of the SG structure to the vessel anatomy, they do not protect from progression of aneurysmal degeneration at the necks and do not promote 
biological fixation of SG into the vessel wall. Indeed, the external cover of those devices is made of PTFE, and the in vitro cell culture tests performed here confirmed once again that this polymer fails to promote the cell-surface interactions that are required to achieve adequate cell adhesion and survival in a pro-apoptotic environment such as AAA. PTFE displays very low surface energy and extreme hydrophobicity [21], and the near-complete lack of cell colonization observed in vitro illustrates the insufficient tissue ingrowth in vivo on ePTFE SG after EVAR [22].

We believe that, in order to prevent late occurrence of endoleaks due to tissue degeneration at the aneurysm necks, it is crucial to achieve a sustained healing in the aneurysm sac and especially at the proximal and distal neck so as to achieve durable biological sealing. For that the SG external surface should favor smooth muscle cell as well as fibroblast adhesion and survival. Indeed, those two cell types play an important role in vascular remodeling following injury [23].

Physico-chemical surface modifications alone do not lead to targeted cell responses as opposed to modifications with biological factors [24]. This was illustrated here, where the amine-rich polymer (LP) increased initial cell adhesion, but was ineffective to achieve adequate cell survival. A coating with tailored bioactive properties is needed to prevent cell apoptosis in the SG landing zones, and possibly to stop the disease progression at the aneurysm neck. Our approach has therefore been to immobilize pro-survival biomolecules, CS and EGF, on the external polymer surface of the SG. 
In vitro results showed significant increase in the survival of both human SMC and fibroblasts. Interestingly and of considerable importance, the present coating maintained its bioactivity over a one-month storage period at various temperatures. The persistence of coating bioactivity is crucial to any clinical application, since a commercial bioactive SG will need to possess relatively long shelf life to accommodate inevitable delays between production and implantation. Moreover, good stability of the biomolecules prevent their leakage and undesirable effects on remote tissues.

In vivo results strongly suggest that our coating improves healing around the SG and minimizes the fresh thrombus and endoleak area, even though the number of animals in our present study was rather limited ( $\mathrm{n}=3$, with 2 aneurysms per animal). No complication or reduction of the SG internal lumen due to neointimal formation was observed, in contrast with other studies, where bioactive SG were found to induce partial stenosis [25]. However, the bioactive coating did not lead to complete healing of the cavity or to total sealing, as initially created endoleaks persisted even with the coated SG.

These results therefore appear mitigated, but they may be explained by the type of model used in this study, probably not the most appropriate to test our hypothesis. The limitations of our model are i) the creation of large endoleaks immediately after implantation; ii) the absence of a pathological vessel in our vein-patch model.

Indeed, large endoleaks were created after SG implantation. The persistent flow into the cavity tended to impede the important interactions between the bioactive coating and 
cells of the surrounding vessel wall. For a potential clinical use of our bioactive SG, in cases presenting a deficient SG apposition, or late occurrence of type II endoleaks coming from collateral arteries, a concomitant strategy could be used, such as inflatable polymeric bags [20] or injection of embolizing gels [26].

Additionally, the vein patch used in this study provides a geometrical aneurysm model, one with realistic flow conditions; however, this model is exempt of atherosclerosis and calcium deposits, it displays normal ECM, without inflammation and SMC depletion, unlike a real AAA [3].

To further evaluate the effect of our present anti-apoptotic bioactive coating on tissue healing after EVAR, our in vivo model should be adapted to mimic damage related to ECM and SMC apoptosis, two key characteristics of AAA, and to suppress large type I endoleaks that have previously been created by deformation of the SG during implantation. To this end, aneurysms may be created using decellularized xenografts [27], elastase infusion, calcium chloride or angiotensin-II perfusion [28].

In conclusion, a bioactive coating based on chondroitin sulfate (CS) and epidermal growth factor (EGF) was shown to increase the survival of human vascular smooth muscle cells and fibroblasts in in vitro pro-apoptotic conditions and to increase healing in canine aneurysms when deposited on the external surface of a commercial SG. While further in vivo testing is required to demonstrate efficacy and to secure regulatory approval, the present results suggest that this bioactive coating may improve healing after 
EVAR and thus have a positive impact on the prevention of complications and aneurysm progression with time.

\section{References}

1. Major, A., et al., Implant degradation and poor healing after endovascular repair of abdominal aortic aneurysms: An analysis of explanted stent-grafts. Journal of Endovascular Therapy, 2006. 13(4): p. 457-467.

2. Malina, M., et al., Endovascular AAA Exclusion: Will Stents With Hooks and Barbs Prevent Stent-Graft Migration? Journal of Endovascular Surgery, 1998. 5(4): p. 310-317.

3. Henderson, E.L., et al., Death of smooth muscle cells and expression of mediators of apoptosis by $T$ lymphocytes in human abdominal aortic aneurysms. Circulation, 1999. 99(1): p. 96-104.

4. Wills, A., et al., Pathogenesis of abdominal aortic aneurysms - Cellular and biochemical mechanisms. European Journal of Vascular and Endovascular Surgery, 1996. 12(4): p. 391-400.

5. Mertens, J., et al., Long-term results after endovascular abdominal aortic aneurysm repair using the Cook Zenith endograft. J Vasc Surg, 2011. 54(1): p. 48-57.e2.

6. Bastos Goncalves, F., et al., Risk factors for proximal neck complications after endovascular aneurysm repair using the endurant stentgraft. Eur $\mathrm{J}$ Vasc Endovasc Surg, 2015. 49(2): p. 156-62.

7. Cao, P., et al., Predictive factors and clinical consequences of proximal aortic neck dilatation in 230 patients undergoing abdominal aorta aneurysm repair with self-expandable stent-grafts1. Journal of Vascular Surgery, 2003. 37(6): p. 12001205.

8. Litwinski, R.A., et al., The role of aortic neck dilation and elongation in the etiology of stent graft migration after endovascular abdominal aortic aneurysm repair with a passive fixation device. Journal of Vascular Surgery. 44(6): p. 11761181.

9. Theocharis, A.D., et al., Compositional and structural alterations of chondroitin and dermatan sulfates during the progression of atherosclerosis and aneurysmal dilatation of the human abdominal aorta. Biochimie, 2002. 84(7): p. 667-674.

10. Raymond, M.A., et al., Apoptosis of endothelial cells triggers a caspasedependent anti-apoptotic paracrine loop active on vascular smooth muscle cells. Faseb Journal, 2004. 18(2): p. U166-U184.

11. Barrientos, S., et al., Growth factors and cytokines in wound healing. Wound Repair and Regeneration, 2008. 16(5): p. 585-601. 
12. Ying, W.Z., H.G. Zhang, and P.W. Sanders, EGF receptor activity modulates apoptosis induced by inhibition of the proteasome of vascular smooth muscle cells. Journal of the American Society of Nephrology, 2007. 18(1): p. 131-142.

13. Njieha, F.K. and S.W. Shalaby, Stabilization of epidermal growth factor. Journal of Bioactive and Compatible Polymers, 1992. 7(3): p. 288-299.

14. Lequoy, P., et al., Additive benefits of chondroitin sulfate and oriented tethered epidermal growth factor for vascular smooth muscle cell survival. Macromol Biosci, 2014. 14(5): p. 720-30.

15. Soulez, G., et al., Type I and Collateral Flow in Experimental Aneurysm Models Treated with Stent-Grafts. Journal of Vascular and Interventional Radiology, 2007. 18(2): p. 265-272.

16. Lerouge, S., et al., Nitrogen-rich coatings for promoting healing around stentgrafts after endovascular aneurysm repair. Biomaterials, 2007. 28(6): p. 12091217.

17. Charbonneau, C., et al., Chondroitin Sulfate and Epidermal Growth Factor Immobilization after Plasma Polymerization: A Versatile Anti-Apoptotic Coating to Promote Healing Around Stent Grafts. Macromolecular Bioscience, 2012. 12(6): p. 812-821.

18. Charbonneau, C., et al., Stimulation of cell growth and resistance to apoptosis in vascular smooth muscle cells on a chondroitin sulfate/epidermal growth factor coating. Biomaterials, 2011. 32(6): p. 1591-1600.

19. Melas, N., et al., Active proximal sealing in the endovascular repair of abdominal aortic aneurysms: early results with a new stent-graft. J Endovasc Ther, 2015. 22(2): p. 174-8.

20. Holden, A., Endovascular sac sealing concept: will the Endologix Nellix device solve the deficiencies? J Cardiovasc Surg (Torino), 2015. 56(3): p. 339-53.

21. Jie-Rong, C. and T. Wakida, Studies on the surface free energy and surface structure of PTFE film treated with low temperature plasma. Journal of Applied Polymer Science, 1997. 63(13): p. 1733-1739.

22. McArthur, C., et al., Histopathologic analysis of endovascular stent grafts from patients with aortic aneurysms: Does healing occur? Journal of Vascular Surgery, 2001. 33(4): p. 733-738.

23. Sartore, S., et al., Contribution of Adventitial Fibroblasts to Neointima Formation and Vascular Remodeling: From Innocent Bystander to Active Participant. Circulation Research, 2001. 89(12): p. 1111-1121.

24. Shen, H., et al., The immobilization of basic fibroblast growth factor on plasmatreated poly(lactide-co-glycolide). Biomaterials, 2008. 29(15): p. 2388-2399.

25. Kusanagi, M., et al., Hydrogel-mediated release of basic fibroblast growth factor from a Stent-Graft accelerates biological fixation with the aortic wall in a porcine model. Journal of Endovascular Therapy, 2007. 14(6): p. 785-793.

26. Fatimi, A., et al., A new injectable radiopaque chitosan-based sclerosing embolizing hydrogel for endovascular therapies. Acta Biomaterialia, 2012. 8(7): p. 2712-2721.

27. Franck, G., et al., Reestablishment of the Endothelial Lining by Endothelial Cell Therapy Stabilizes Experimental Abdominal Aortic Aneurysms. Circulation, 2013. 127(18): p. 1877-1887. 
28. Trollope, A., et al., Animal models of abdominal aortic aneurysm and their role in furthering management of human disease. Cardiovascular Pathology, 2011. 20(2): p. 114-123.

\section{Supplemental figures}

a.

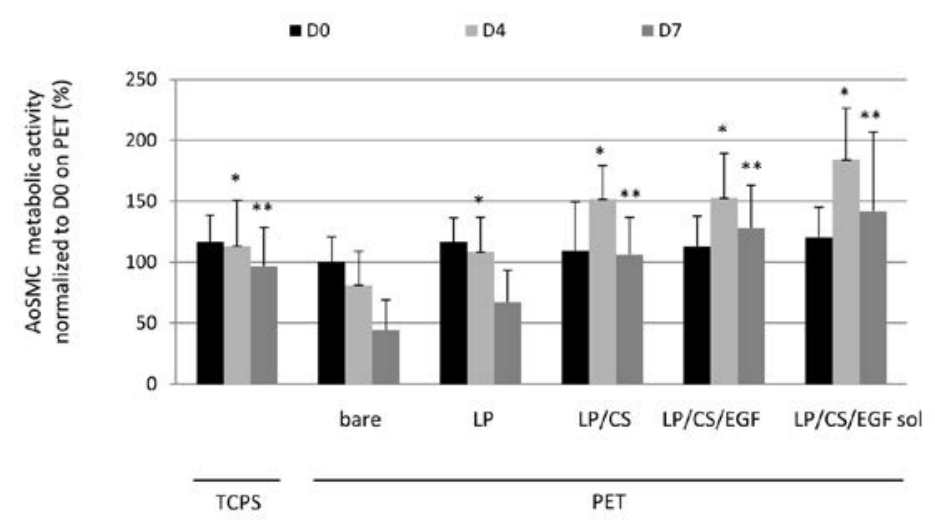

b.

TCPS

PET

LP

LP/CS

LP/CS/EGF

LP/CS/EGFsol

Do
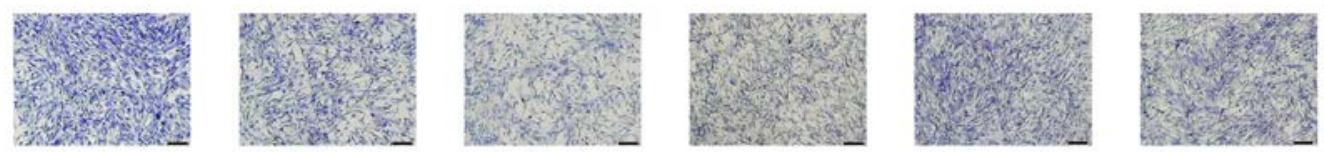

D7
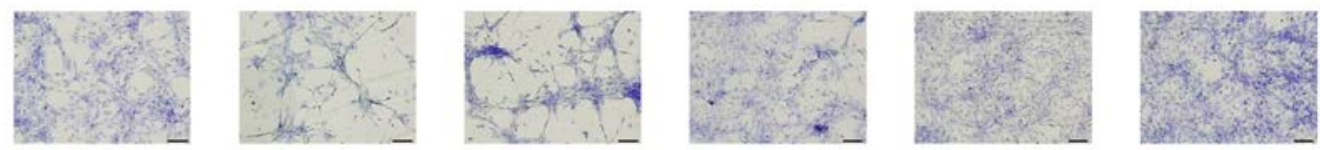

Figure E1. SEM images displaying the surface morphology of ePTFE before (a) and after bioactive coating creation (b). Scale bars: 25 $\mu \mathrm{m}$. ePTFE: expanded polytetrafluoroethylene, bioactive coating: primary amine-rich plasma polymer, chondroitin sulfate and epidermal growth factor coating (LP/CS/EGF). SEM observations showed no damage or changes in porosity after applying the bioactive coatings, compared with bare ePTFE. 
a.

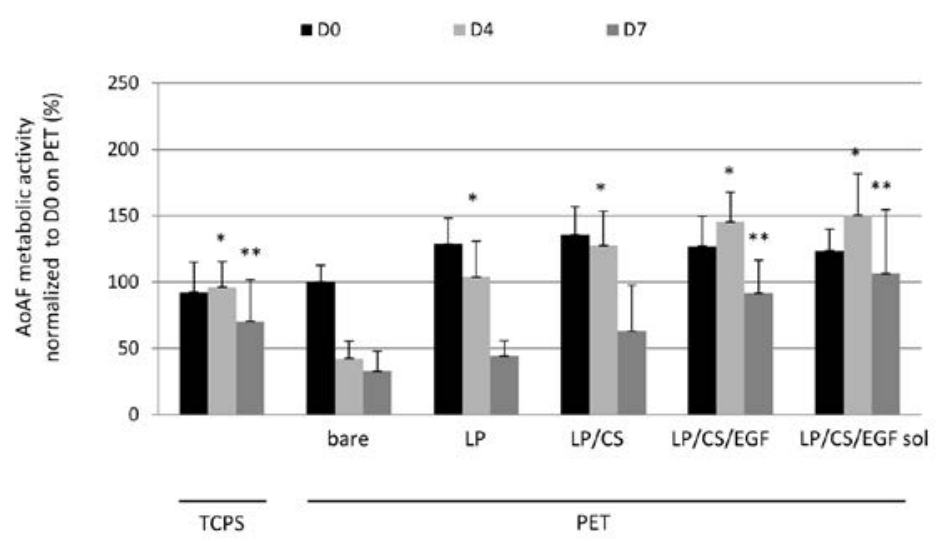

b.
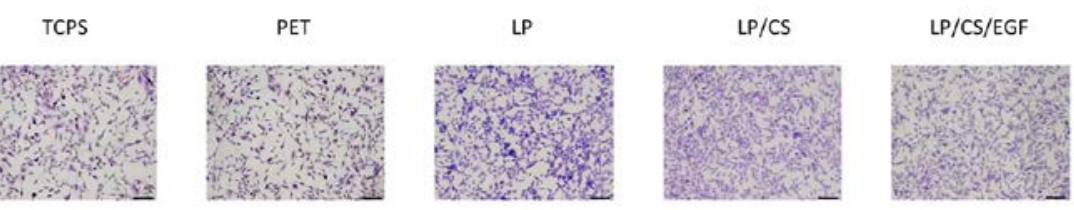

LP/CS/EGF sol

D7
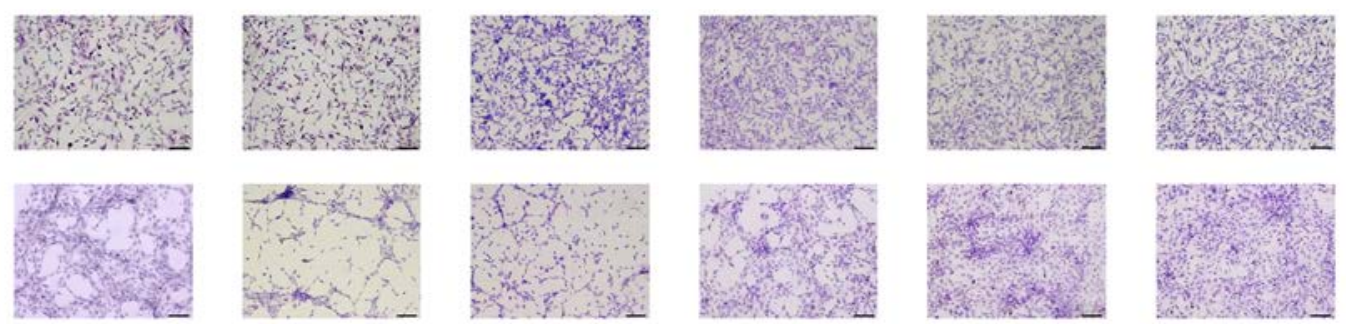

Figure E2. Stress-strain curve of ePTFE with and without the bioactive coating (average calculated with $n=6$ for each condition). ePTFE: expanded polytetrafluoroethylene, LP: primary amine-rich plasma polymer coating, LP/CS/EGF: primary amine-rich plasma polymer, chondroitin sulfate and epidermal growth factor coating. The mechanical properties of ePTFE were conserved after LP deposition and successive biomolecule grafting steps. 

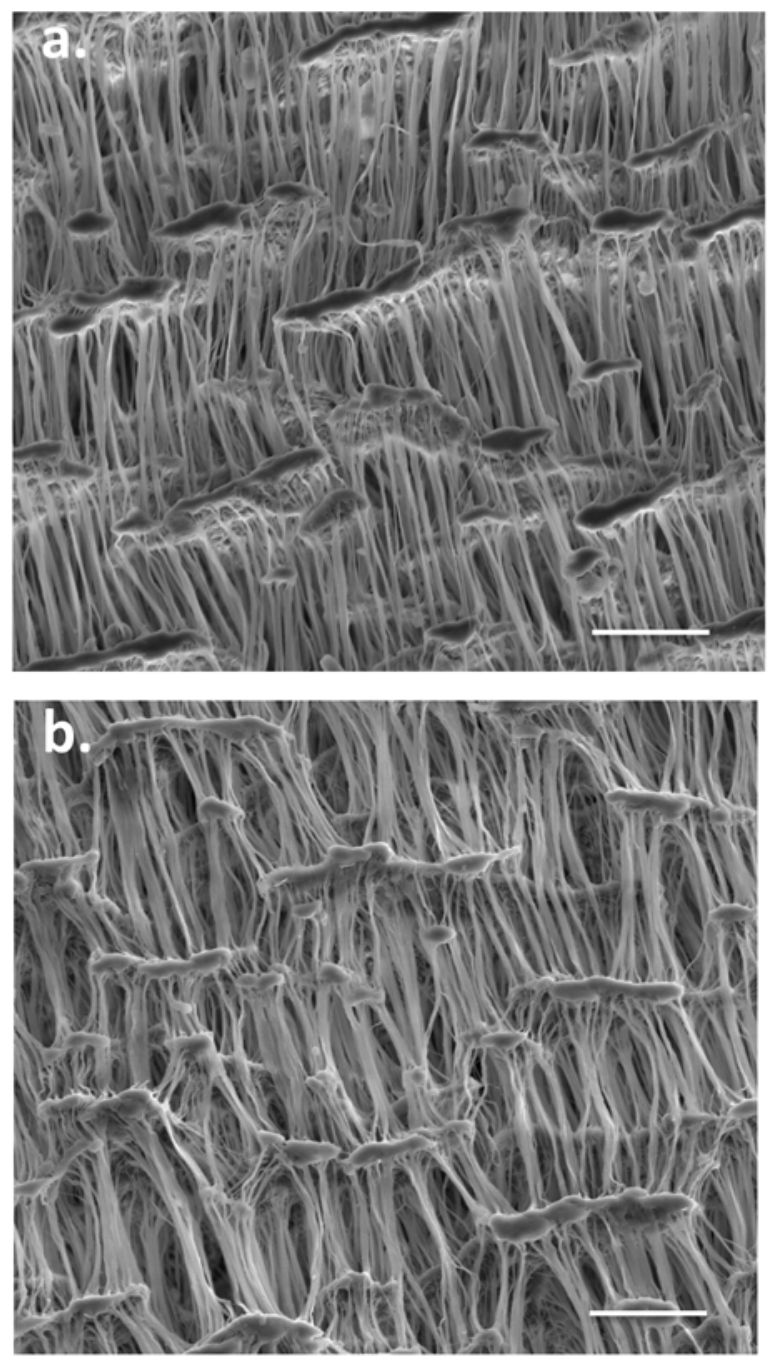

Figure E3. Human aortic smooth muscle cells adhesion (D0) and survival on bioactive and control PET when immersed in serum free medium for 4 and 7 days (D4, D7), estimated by alamarBlue (a) and observed by cristal violet staining (b). Scale bars: $200 \mu m$. The bioactive coating (LP/CS/EGF) led to a significant increase in SMC survival over 4 and 7 days, compared with bare PET. ${ }^{*}, * *: P<.05$ compared to PET at D4 and D7 respectively ( $n=12)$. TCPS: tissue culture polystyrene, PET: polyethylene terephtalate film, LP: primary amine-rich plasma polymer coating, CS: chondroitin sulfate coating, EGF: epidermal growth factor coating, EGF sol: epidermal growth factor added in solution at $10 \mathrm{ng} / \mathrm{ml}$. 


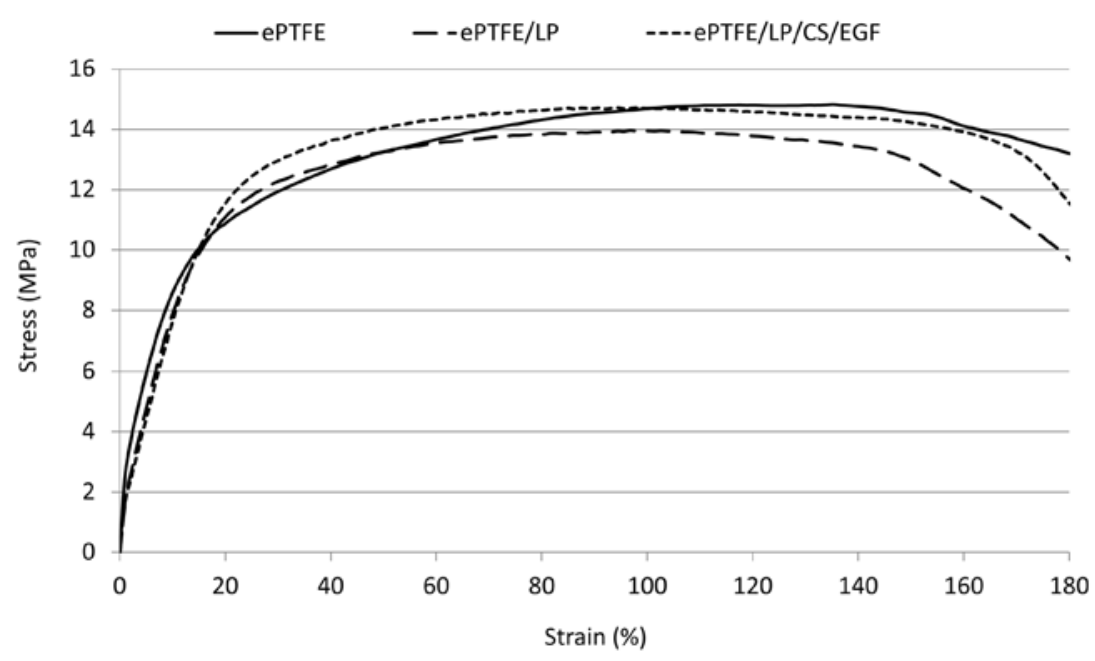

Figure E4. Human aortic fibroblasts adhesion (D0) and survival on bioactive and control PET surfaces when immersed in serum free medium for 4 and 7 days (D4, D7), estimated by alamarBlue (a) and observed by cristal violet staining (b). Scale bars: $200 \mu \mathrm{m}$. Coating with LP/CS/EGF led to a significant increase in fibroblast survival over 4 and 7 days, compared with bare PET. *, **: $P<.05$ compared to PET at D4 and $D 7$ respectively $(n=12)$. TCPS: tissue culture polystyrene, PET: polyethylene terephtalate film, LP: primary amine-rich plasma polymer coating, CS: chondroitin sulfate coating, EGF: epidermal growth factor coating, EGF sol: epidermal growth factor added in solution at $10 \mathrm{ng} / \mathrm{ml}$.
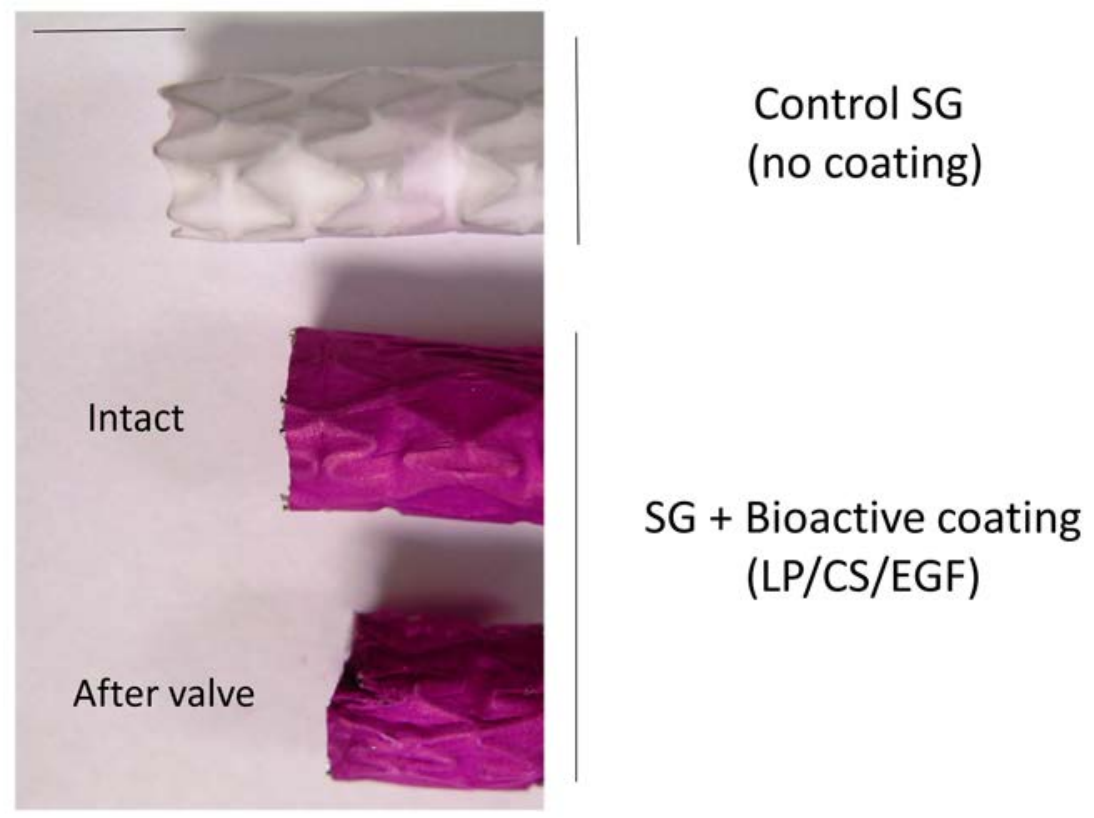

Figure E5. Coated SG before and after passage through an insertion valve. Coating is stained using fuchsine acid (0.35\% w/v solution in 33\% v/v acetic acid). Scale bar: $5 \mathrm{~mm}$. LP/CS/EGF: primary amine-rich plasma polymer, chondroitin sulfate and epidermal 
growth factor coating. The polymer coating (LP) was homogeneous over the entire SG surface, even after passage through the hemostasis valve of the introducer system.

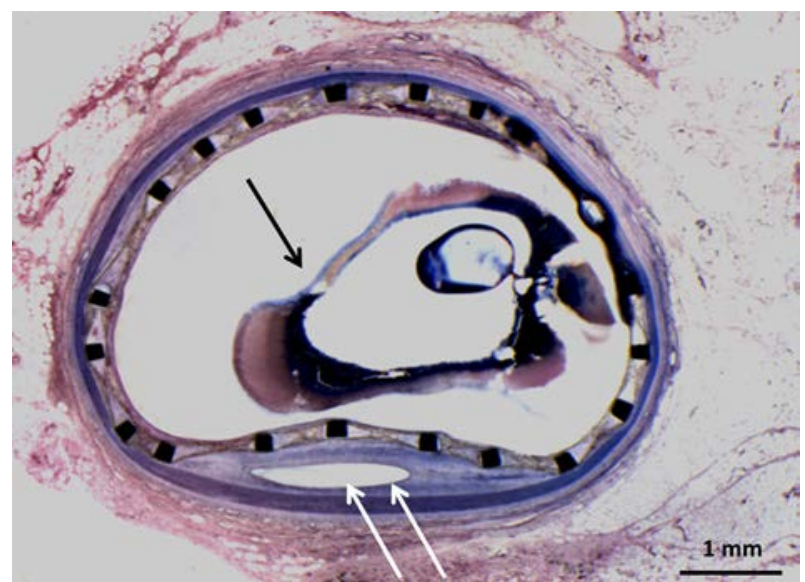

Figure E6. Exakt histological section (with hematoxylin and eosin staining) of an explanted SG showing the misfit created with a balloon catheter after implantation at the proximal neck to create a type I endoleak (double white arrow). Scale bar: $1 \mathrm{~mm}$. n.b some post-mortem intraluminal thrombus is present in the lumen (single black arrow). 